\title{
Synergistic antitumor effects of liposomal honokiol combined with cisplatin in colon cancer models
}

\author{
NIANG CHENG ${ }^{*}$, TIAN XIA* ${ }^{*}$ YING HAN, QING JUN HE, RONG ZHAO and JUN RONG MA \\ Clinical Skills Training Center, West China Hospital, West China Medical School, \\ Sichuan University, Chengdu, Sichuan 610041, P.R. China
}

Received February 23, 2011; Accepted June 15, 2011

DOI: $10.3892 / \mathrm{ol} .2011 .350$

\begin{abstract}
Honokiol, a novel antitumor agent, may induce apoptosis and inhibit the growth of vascular endothelium in a number of tumor cell lines and xenograft models. It has been proposed that the antitumor effects of chemotherapy may be increased in combination with an antiangiogenesis agent as an anticancer strategy. In the present study, we examined the potential of honokiol to increase the antitumor effect of cisplatin (DDP) when the agent and drug were combined in murine CT26 colon cancer models, and investigated the underlying mechanism. Liposomal honokiol (LH) was prepared, and female BALB/c mice were administered $\mathrm{LH}$ at various doses to determine the optimum doses for honokial. Evaluation of cell apoptosis was analyzed using flow cytometry. Honokiol was encapsulated with liposome to improve its water insolubility. In vitro, LH inhibited the proliferation of CT26 cells via apoptosis and significantly enhanced the DPP-induced apoptosis of CT26 cells. In vivo, the systemic administration of LH plus DDP resulted in the inhibition of subcutaneous tumor growth beyond the effects observed with either LH or DDP alone. This growth reduction was associated with elevated levels of apoptosis (TUNEL staining) and reduced endothelial cell density (CD31 staining) compared with either
\end{abstract}

Correspondence to: Dr Tian Xia, Clinical Skills Training Center, West China Hospital, West China Medical School, Sichuan University, 37 Guoxuexiang Lane, Wuhou District, Chengdu, Sichuan 610041, P.R. China

E-mail: summer@cd120.com

*Contributed equally

Abbreviations: CI, combination index; DDP, cisplatin; EL, empty liposomes; IC50, half maximal inhibition concentration; LH, liposomal honokiol; MTT, 3-(4,5-dimethylthiazol-2-yl)-2,5diphenyltetrazolium bromide; MVD, microvessel density; NS, normal saline; PC, phosphatidylcholine; PEG, polyethylene glycol; PI, propidium iodide; TUNEL, TdT-mediated dUTP nick-end labeling; VEGF, vascular endothelial growth factor; VEGFR, VEGF receptor

Key words: honokiol, liposome, cisplatin, apoptosis, antiangiogenesis treatment alone. Collectively, these findings indicate that LH may augment the induction of apoptosis in CT26 cells in vitro and in vivo, and this combined treatment has exhibited synergistic suppression in tumor progression according to the synergistic analysis. The present study may be significant to future exploration of the potential application of the combined approach in the treatment of colon cancer.

\section{Introduction}

Systemic therapy of cancer has been dominated by chemotherapy. Cisplatin (DDP), a platinum-containing anticancer drug, remains the most widely used first-line element of cytotoxic agents for the treatment of solid malignant tumors $(1,2)$. However, DDP-based regimens often cause severe toxic side effects such as myelosuppression, asthenia and gastrointestinal disorder, as well as long-term cardiac, renal and neurological consequences. These adverse events usually cause drug discontinuation, poor tolerance and limited therapeutic efficacy $(3,4)$. One promising strategy to reduce the toxicity of chemotherapy while maintaining its therapeutic effects is the combination of low-dose conventional chemotherapeutic drugs with agents that are likely to increase cellular chemosensitivity (5).

Honokiol, an active compound purified from magnolia, has drawn much attention for its antiangiogenesis and antitumor properties. Findings of previous studies showed that honokiol induces apoptosis in a variety of cancer cell lines, including murine endothelial SVR cells (6), human leukemia MOLT 4B cells (7), human colorectal carcinoma RKO cells (8) and human squamous lung cancer $\mathrm{CH} 27$ cells (9). Additionally, honokiol may inhibit the growth of new vessels by interfering with the phosphorylation of vascular endothelial growth factor 2 (VEGF2), and has been found to induce apoptosis of aggressive angiosarcoma in nude mice $(6,10)$. These findings indicate that honokiol possesses antiangiogenic and antitumor activities. Studies pertaining to honokiol's in vivo antitumor activity against skin tumors and SVR angiosarcoma in a mouse model are available $(6,11)$. However, little is known about the antitumor activity of honokiol in colorectal cancer in animal models.

Due to the differences between their possible mechanisms of action and toxicity profiles, the combination of honokiol and DDP may have clinical potential. Therefore, the present study 
was designed to determine whether liposomal honokiol (LH) is capable of antitumor activity against a mouse CT26 cell line, and whether it is capable of synergistically potentiating the antitumor activity of DDP in the BALB/c mice bearing CT26 cell line xenografts in vivo. The combined mechanisms underlying the antitumor effects were investigated by observing the microvessel density (MVD) and apoptosis in tumor tissues.

\section{Materials and methods}

Reagents. High-purity soybean phosphatidylcholine (PC), polyethylene glycol (PEG) 4000, and cholesterol were purchased from Sigma (St. Louis, MO, USA). Honokiol was obtained from Chengdu Sikehua Biotechnology Co. (Chengdu, China). DDP was purchased from QiLu Pharmaceutical Co. (Shandong, China). A rat antimouse CD31 monoclonal antibody was purchased from BD Biosciences Co. (Pharmingen, San Diego, CA, USA). An in situ cell death detection kit was purchased from Roche Applied Sciences (Indianapolis, IN, USA).

Cell lines and cell culture. The murine colon carcinoma cell line CT26 was obtained from the American Type Culture Collection. The cells were grown as monolayers in RPMI-1640 (Gibco), containing 10\% heat-inactivated fetal calf serum, $100 \mathrm{U} / \mathrm{ml}$ penicillin and $100 \mathrm{U} / \mathrm{ml}$ streptomycin, at $37^{\circ} \mathrm{C}, 95 \%$ relative humidity, under $5 \% \mathrm{CO}_{2}$.

Preparation of $\mathrm{LH}$. LH was prepared in our laboratory as follows: cholesterol, PEG 4000 and honokiol in weight ratios of 0.15:0.24:0.22 were mixed and dissolved in $15 \mathrm{ml}$ chloroform/methanol at a ratio of 1:4 (v/v). The mixture was gently warmed to $40^{\circ} \mathrm{C}$ in a round-bottomed flask, and evaporated under vacuum in a rotary evaporator until a thin lipid film was formed. The dried lipid films were left overnight and sonicated in double distilled $\mathrm{H}_{2} \mathrm{O}$, and then concentrated and lyophilized. Empty liposomes (EL) were prepared in the same manner as LH, without the addition of honokiol. The final LH and EL were small multilamellar liposomes at a size range of $120 \pm 20$ and $90 \pm 20 \mathrm{~nm}$, respectively. Lyophilized $\mathrm{LH}$ and EL were dissolved in $5 \%$ glucose for in vitro and in vivo studies.

Apoptosis assay in vitro. Aliquots of $1 \times 10^{5}$ CT26 cells were grown in each well of 6-well plates in triplicate and incubated for $72 \mathrm{~h}$ until $80 \%$ confluence was achieved. For DDP, the IC50 (48 h) value of CT26 cells was $10 \mu \mathrm{M}$. For $\mathrm{LH}$, the IC50 (48 h) value of CT26 cells was $36 \mu \mathrm{M}$ (MTT assay). Following $48 \mathrm{~h}$ of incubation, quantitative evaluation of cell apoptosis was performed by flow cytometry using the annexin V/propidium iodide (PI) double-staining method. Briefly, after processing in 6-well plates as described above, the floated cells were discarded and the attached cells were trypsinized and washed twice with cold phosphatebuffered saline (PBS). Cells were resuspended in prediluted binding buffer and stained with FITC-labeled annexin V (BD Pharmingen, CA, USA) for $10 \mathrm{~min}$ in the dark at room temperature. The cells were then washed and resuspended in binding buffer. PI $(1 \mu \mathrm{g} / \mathrm{ml})$ was added, and the mixtures were analyzed immediately by flow cytometry.
Murine tumor model and treatment. Female BALB/c mice aged 6-8 weeks were purchased from the Laboratory Animal Center of Sichuan University, China, and allowed to acclimate for 1 week prior to use. Studies involving mice were performed in accordance with institutional guidelines concerning laboratory animal care and use. In our preliminary investigation, we performed a series of experiments in order to determine the optimum doses for the two agents. Murine CT26 colon cancer cells $\left(1.0 \times 10^{7}\right)$ were injected subcutaneously. Once tumors were palpable, mice were randomly assigned into each group ( $n=5$ mice/group). Treatment was administered in the form of intravenous injection with PBS, liposome, free honokiol alone, or LH at different doses of 1 to $50 \mathrm{mg} / \mathrm{kg}$ every day for 21 days, respectively. In the following set of experiments, approximately $5 \times 10^{5} \mathrm{CT} 26$ colon cancer cells were inoculated subcutaneously in the right flank of each mouse. Mice were randomly divided into five groups when the size of tumors reached approximately $30 \mathrm{~mm}^{3}$ ( 6 days after tumor cell inoculation), and received the corresponding treatment: normal saline (NS) $(0.2 \mathrm{ml}$ each), EL (30 mg/kg), LH (25 mg/kg), DDP (5 mg/kg, in $0.2 \mathrm{ml} \mathrm{NS})$, and LH (25 mg/kg) plus $5 \mathrm{mg} / \mathrm{kg}$ DDP. Each group of mice was given treatment via the tail vein on days $6,11,16$ and 21 after tumor cell inoculation. The regime of DDP (QiLu Pharmaceutical Co.) was cycled twice on days 6 and 12 after the initiation of treatment. The experiment for the observation of tumor volume included 10 mice per group. Two perpendicular diameters of tumors were measured every 2 days with a caliper square by the same investigator. The mice were sacrificed when they became moribund. To detect the MVD and apoptosis, excised tumor tissues were fixed in $10 \%$ formalin. Mice were also monitored for possible side effects, including loss of weight or appetite or altered behavior, during the treatment.

Synergistic analysis. At the endpoint of treatment, tumor regression was calculated using the formula: tumor suppression index $=\left(\mathrm{V}_{0}-\mathrm{V}\right) / \mathrm{V}_{0} \times 100 \%$, where $\mathrm{V}$ is the mean tumor volume of the treated group, and $\mathrm{V}_{0}$ is the mean tumor volume of the control group. In each group, the relative tumor volume was expressed as the ratio $\mathrm{V}_{\mathrm{t}} / \mathrm{V}_{0}$ where $\mathrm{V}_{\mathrm{t}}$ is the mean tumor volume on a given day during the treatment and $\mathrm{V}_{0}$ is the mean tumor volume at the commencement of the treatment. The expected reduced percentage of tumor volume (Rptv) of the combined treatment was calculated according to the following modified Jin's formula (12): expected reduced percentage of tumor volume $=\operatorname{drug} \mathrm{A}+(1-\operatorname{drug} \mathrm{A}) \mathrm{x} \operatorname{drug} \mathrm{B}$ or drug $\mathrm{B}+(1-\operatorname{drug} \mathrm{B}) \mathrm{x} \operatorname{drug} \mathrm{A}$. The combination index $(\mathrm{CI})$ was assessed by the ratio of the actual Rptv divided by that of the expected Rptv.

If CI was $<0.85$, the combination was considered antagonistic; at $>1.15$ the combination was considered synergistic; and where $0.85 \leq \mathrm{CI} \leq 1.15$, the combination was considered additive.

TUNEL assay. Apoptotic cells were identified by fluorescent in situ terminal deoxynucleotidyl transferase-mediated dUTP nick-end labeling (TUNEL) staining (in situ cell death detection kit, Roche) according to the manufacturer's instructions. Images were captured by fluorescence microscopy at an excitation wavelength of $488 \mathrm{~nm}$. The apoptotic cells were 
A

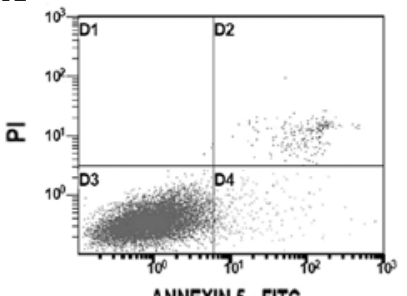

ANNEXIN 5 - FITC

C

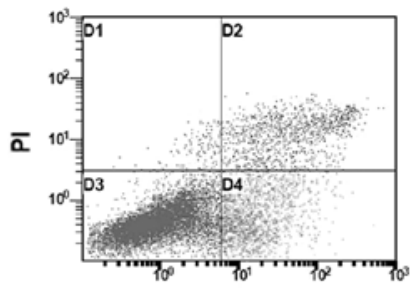

ANNEXIN 5 - FITC

$\mathbf{E}$

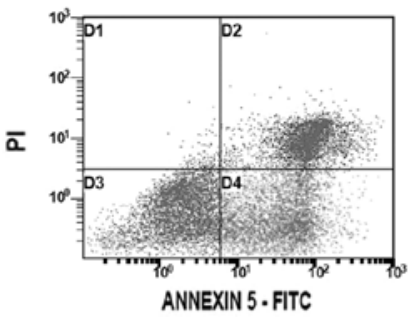

B

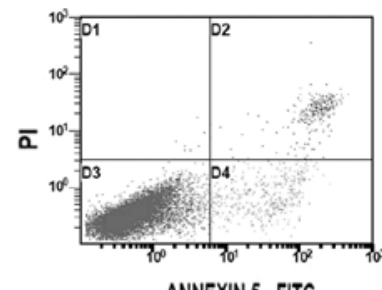

ANNEXIN 5 - FITC

D

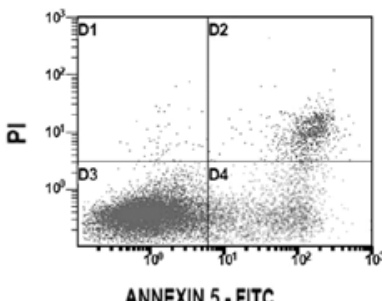

considered to be statistically significant. Results are shown as the means \pm SD. Experiments were performed at least in duplicate.

\section{Results}

LH enhancement of the in vitro antitumor effects of DDP. Flow cytometry of the annexin V/PI double-staining method was used to evaluate the effect of LH and DDP on the induction of apoptosis in CT26 cells. Both early and late apoptotic cells were represented by annexin V-positive and PI-negative or positive cells (right quadrants). The LH+DDP (61.39\%) treatment significantly increased the number of apoptotic cells compared with the other treatments (NS, 3.17\%; EL, 4.5\%; LH, 20.33\% and DDP, 21.14\%) $(\mathrm{P}<0.05)$ (Fig. 1). The interactive antitumor effects of combined treatment with $\mathrm{LH}$ and DDP were greater than the additive when compared with the expected effects of combined treatment in vitro. The expected combined apoptotic effect was defined as: expected combined effect $=$ LH effect + DDP effect - LH effect $x$ DDP effect. The LH treatment induced a CT26 cell apoptosis of $20.33 \%$ and the DDP treatment induced a CT26 cell apoptosis of $21.14 \%$. Based on these values, the expected $\%$ reduction in the combined treatment group should have been $31.17 \%$. However, the $\%$ reduction in the combined treatment group was $61.39 \%$, indicating synergistic effects.

Immunohistochemistry. The antiangiogenetic effects of LH plus DDP were determined by CD31 immunostaining. Paraffinembedded tumor sections were probed with a monoclonal rat anti-mouse CD31 antibody (1:400) at $4^{\circ} \mathrm{C}$ overnight, followed by incubation with biotinylated polyclonal goat anti-rat antibody (1:200) in a humidified chamber for $1 \mathrm{~h}$. The sections were then immersed in $0.3 \% \mathrm{H}_{2} \mathrm{O}_{2}$ in absolute methanol for 15 min to block endogenous peroxidase. A positive reaction was visualized using chromagen 3,3'-diaminobenzidine (DAB substrate kit). Sections were counterstained with hematoxylin and mounted with glass coverslips. Tissue sections were visualized using an Olympus microscope at a magnification of x400 to determine the MVD.

Statistical analysis. Statistical tests were performed using SPSS 13.0 software. Statistical comparisons were made with single factor analysis of variance with the Student-NewmanKeuls (SNK) test used for post hoc comparisons. $\mathrm{P}<0.05$ was

Tumor growth inhibition. To determine the optimum doses for honokiol, human CT26-bearing BALB/c mice were treated with $\mathrm{LH}$ at various doses ranging from 1 to $50 \mathrm{mg} / \mathrm{kg}$. The mice treated with 15 and $10 \mathrm{mg} / \mathrm{kg} \mathrm{LH}$ exhibited different inhibiting responses to the tumor compared with the control group, including the free liposome group and PBS controls. When the dose of LH was elevated to $25 \mathrm{mg} / \mathrm{kg}$, tumor growth was significantly inhibited, whereas no further enhancement of the antitumor activity occurred at a dose of $50 \mathrm{mg} / \mathrm{kg}$. Thus, we selected the dose of $25 \mathrm{mg} / \mathrm{kg}$ as the optimum dose for the combination treatment with DDP.

In the following set of experiments, mice were treated with intravenous injection of $\mathrm{LH}$ at $25 \mathrm{mg} / \mathrm{kg}$ on days 6,11 , 16 and 21 following tumor cell inoculation and/or intravenous administration of DDP on days 6 and 12 after the initiation of treatment or appropriate controls $(0.2 \mathrm{ml}$ PBS or liposome) at 
A

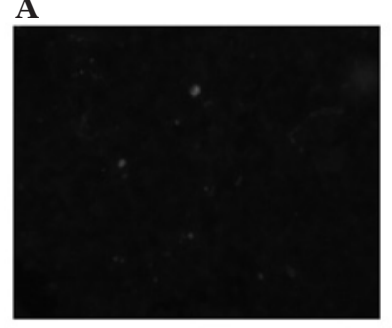

C

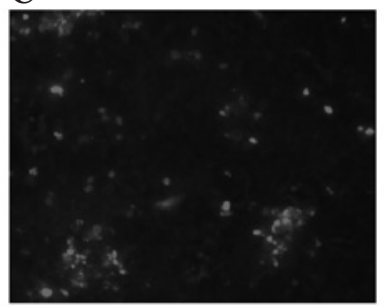

E

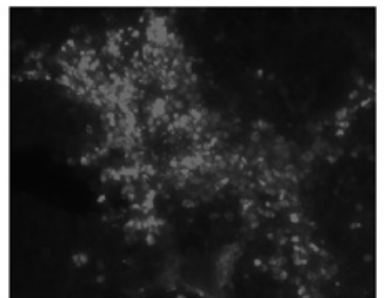

B

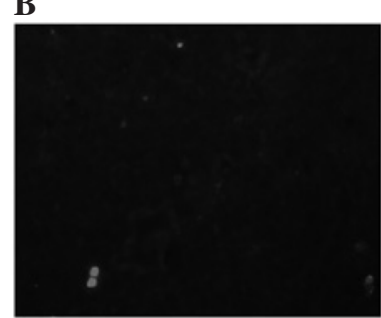

D

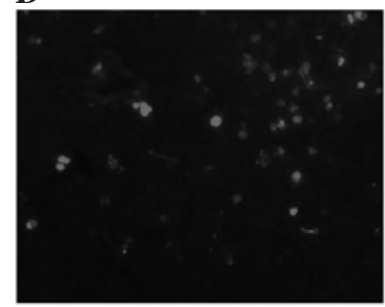

$\mathbf{F}$

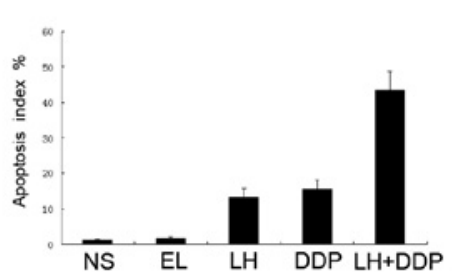

Figure 3. Apoptosis assays of tumor tissues in different groups. (A-E) TUNEL staining of tumors revealed that combination therapy induced a significant enhancement of apoptotic cells in contrast to control therapies; magnification, $x 400$. Apoptosis index of LH plus DDP-treated tumor was elevated compared to that of $(F)$ the control groups. Values are the means \pm SD. LH, liposomal honokiol; DDP, cisplatin; NS, normal saline and EL, empty liposomes.

the same time point. Tumor volumes of mice revealed that the combination group exhibited more effective suppression of tumor growth, whereas LH or DDP administered alone exhibited moderate antitumor efficacy. After 32 days, the average tumor volumes in mice treated with NS, EL, LH, DDP and combination therapy were $4684.85 \pm 1095.79,4433.56 \pm 765.92$, $1813.87 \pm 412.86,2338.24 \pm 531.29$ and $501.03 \pm 263.80 \mathrm{~mm}^{3}$, respectively $(\mathrm{P}<0.05)$ (Fig. 2). The antitumor effects of this combined therapy of LH and DDP revealed that LH synergistically (CI $>1.15$ ) enhanced the antitumor efficacy of DDP.

In vivo induction of apoptosis with the combined treatment. To investigate the role of $\mathrm{LH}$ on the apoptosis of tumor cells, we examined the apoptosis-related molecular marker on tumor sections. An apoptosis detection kit was used to detect early DNA fragmentation associated with apoptosis. The data revealed no significant differences in the apoptosis count between the $0.2 \mathrm{ml} \mathrm{NS}$ (Fig. 3A) and $30 \mathrm{mg} / \mathrm{kg} \mathrm{EL}$ (Fig. 3B) groups. On the other hand, more apoptotic cells were observed in the groups administered with $5 \mathrm{mg} / \mathrm{kg}$ DDP (Fig. 3C) or $25 \mathrm{mg} / \mathrm{kg}$ dose LH (Fig. 3D) alone. Furthermore, tumors from animals receiving $25 \mathrm{mg} / \mathrm{kg} \mathrm{LH}$ plus $5 \mathrm{mg} / \mathrm{kg}$ DDP (Fig. 3E) exhibited the highest apoptotic rate compared with the control group. Fig. 3 shows the synergistic tumor apoptosis effect of LH combined with DDP. Data are the mean apoptotic index \pm SD of cancer cells (Fig. 3F) as \% normalized to the apoptotic index of cancer cells.
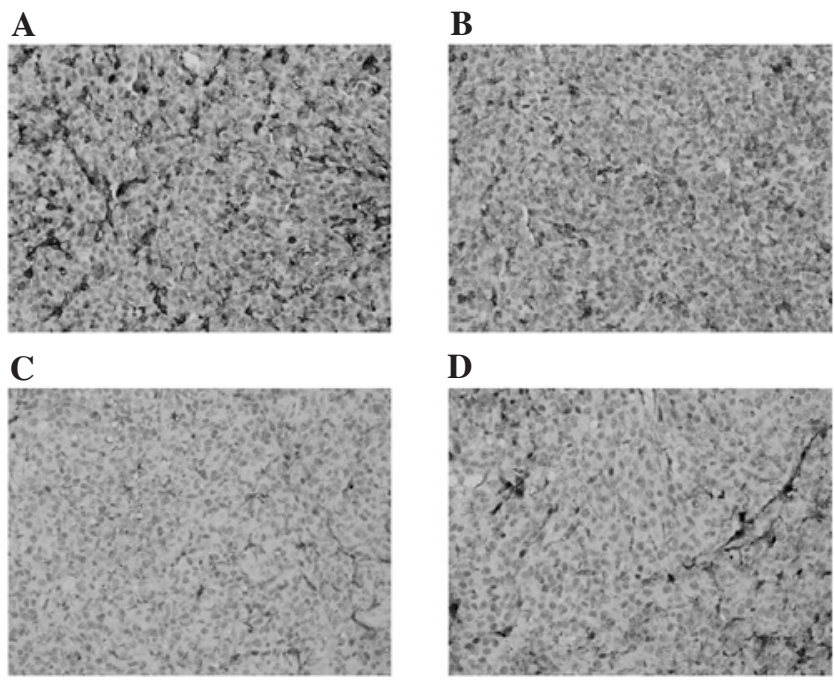

D

$\mathbf{E}$
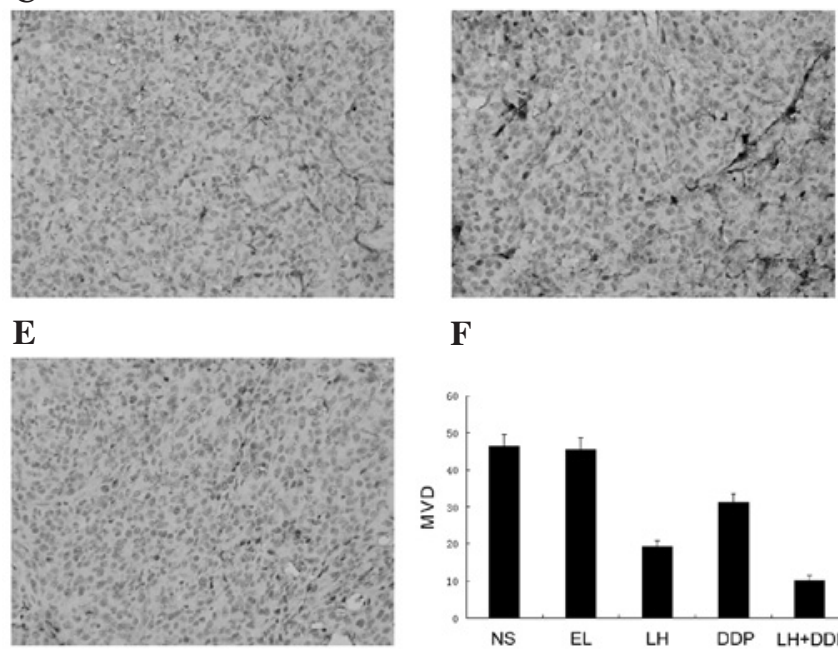

$\mathbf{F}$

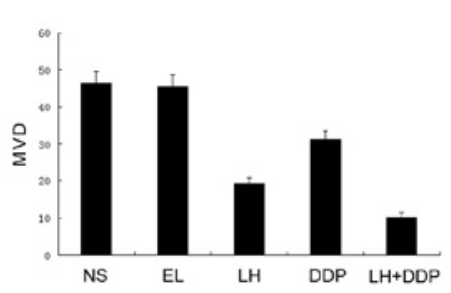

Figure 4. Tumor angiogenesis was assessed by immunohistochemical staining with anti-CD31 antibody on paraffin-embedded tumor sections. MVD counting was performed at a magnification of x400. Significantly reduced numbers of blood vessels in tumors treated with (E) LH plus DDP, in comparison with (A) NS, (B) EL, (C) LH, (D) DDP. (F) The effect of combined therapy on MVD is shown. Vessel density was determined by counting per high-power (F) column, the mean of the microvessels per high-power field; bars, means \pm SD. LH, liposomal honokiol; DDP, cisplatin; NS, normal saline; EL, empty liposomes and MVD, microvessel density.

Inhibition of angiogenesis by the combination of LH plus DDP. Having observed antitumor activity in the CT26 murine colon cancer model, we then quantified vessel density as measures of angiogenesis by immunolabeling CD31 in tissue sections. The combination of LH and DDP (Fig. 4E) appeared to reduce the number of vessels compared with the control groups (Fig. 3), including NS (Fig. 4A), liposome (Fig. 4B), LH (Fig. 4C) and DDP alone (Fig. 4D) $(\mathrm{P}<0.05)$. Angiogenesis was also inhibited in the treatment with honokiol or DDP alone (Fig. 4).

Observation of the potential toxicity. All of the mice exhibited satisfactory drug tolerance and no gross signs of cumulative adverse results, such as weight loss, ruffling of fur or behavioral and postural changes were observed. Furthermore, results from the microscopic examination showed no pathologic changes in the heart, lung, liver, spleen or kidney.

\section{Discussion}

The majority of colon cancer patients are diagnosed at late stages of the disease and have poor prognosis. Clinical outcomes have reached a plateau with conventional chemotherapy as the main treatment of choice. In such clinical settings, an aggressive regimen of chemotherapy may not 
only fail to promote survival, but may also adversely affect quality of life; thus, the mortality rate of this malignancy remains high. Findings of previous studies have shown that drug resistance to chemotherapy is partly mediated by the ability to circumvent apoptosis (13). If chemotherapyinduced DNA damage accumulates beyond a threshold, programmed cell death is likely to be initiated (14).

As is increasingly evident, therapies based on the combination of agents impairing different molecular pathways that regulate tumor cell survival can result in an improvement of current cancer management $(15,16)$. Previous evidence has indicated that honokiol directly triggered apoptosis in a variety of human cancer cells by a number of possible mechanisms: i) suppression of NF-kB-regulated anti-apoptotic signaling (17); ii) activation of caspase-8 and triggering caspase in the tumor necrosis factor apoptotic pathway (18); iii) promotion of the c-Jun $\mathrm{N}$-terminal kinase signaling pathway (19); iv) accumulation of reactive oxygen species (20); v) inhibition of p38 MAPK activation (21); and vi) inhibition of protein synthesis (22), which is entirely distinct from the mechanisms of DDP (23). In this regard, we postulated that honokiol may enhance DDP-induced apoptosis and evaluated the therapeutic efficacy of the combination of LH and DDP on tumor growth in colorectal cancer models.

To the best of our knowledge, the present study has demonstrated for the first time that LH potentiated the therapeutic index of DDP in the treatment of a CT26 tumor-bearing mouse model. The combination treatment of LH plus DDP resulted in synergistic and significant antitumor activity in vitro and in vivo, without obvious systemic toxicity. Although the exact mechanism by which the combination of honokiol with DDP enhanced the therapeutic effects has yet to be fully elucidated, the enhanced antitumor efficacy in vivo may be attributed to increased induction of apoptosis and decreased angiogenesis. This hypothesis is supported by our findings. More apoptotic cells and a lower microvessel count were observed in the tumors of the mice treated with $\mathrm{LH}$ plus DDP than with either agent alone. The first mechanism is increased induction of apoptosis. The combination therapy exhibited a dose and time-dependent inhibition of CT26 cell proliferation, and significantly decreased the IC50 of DDP when combined with LH (data not shown). In vitro combination treatment with LH and DDP significantly increased the apoptosis of colorectal CT26 cancer cells compared with other therapies analyzed by flow cytometry (Fig. 1). The more apparent apoptotic cells were observed in the tumor tissues of the LH+DDP combination-treated group (Fig. 3). The cytotoxicity of LH and DDP exhibit a synergistic relationship with regard to the induction of apoptosis. Our findings were consistent with the apoptosis-inducing ability of honokiol reported previously. The second mechanism is decreased angiogenesis. This mechanism is significant, in light of a study that demonstrated that honokiol may be a potent inhibitor of angiogenesis and may inhibit angiogenesis by interfering with phosphorylation of VEGF receptor 2 (VEGFR2) in human endothelial cells (6). In addition, invasion (MMP-9, IAM-1) and angiogenesis (VEGF) were reported to be down-regulated by honokiol (25). Additionally, it has been reported that DNA synthesis of human umbilical endothelial cells was inhibited in vitro by DDP in a dose-dependent manner, and that rabbit corneal neovascularization in vivo was also suppressed by intravenous injection of DDP, indicating that DDP may have an indirect anti-neoplastic effect through the suppression of neovascularization required for tumor growth (24). In an effort to examine the effect of combination treatment on antiangiogenesis based on the above mechanism, we performed immunohistochemistry for CD31 and calculated MVD. It was revealed that in our experiments, the synergistic effect of combination treatment resulted in a reduction of mean MVD in tumor tissue (Fig. 4), which may contribute to enhanced tumor regression compared with either treatment alone.

The extreme water insolubility of honokiol hampers its delivery to the tumor via intravenous injection. To resolve this problem, a suitable drug delivery system with the aim of making the agent soluble and enhancing its accumulation at the tumor site is required. The most favorable candidate is liposome. Liposome is a widely used vehicle for therapeutic agents, including drugs and genes, in attempts to direct active agents to tumors in vivo $(26,27)$ or to protect sensitive tissues from toxicity (28). It provides slow release of an encapsulated drug, resulting in sustained exposure to tumor cells and enhanced efficacy (29). It accumulates preferentially at tumor sites as a result of its ability to extravasate through 'pores' or 'defects' which are typically in the size range of $400-600 \mathrm{~nm}$ in the capillary endothelium. The 'pores' appear to be a consequence of the rapid angiogenesis occurring in tumors and are generally not present in normal tissues or organs (30). Furthermore, when combined with a hydrophilic coat such as PEG, which is devoid of any specific ligand, liposome accumulates in solid tumors when administered intravenously as a result of a 'leaky' microvasculature and impaired lymphatics in such tumors (31). In light of these characteristics, in the present study, honokiol was encapsulated as a PEG-phosphatidylethanolamine-modified liposome nanoparticle, since the honokiol-encapsulated liposome has exhibited improvement of water solubility. Moreover, our experiments demonstrated that there are no significant differences in tumor growth between EL and NS control groups, and no visible side effects of liposome were observed. Thus, the use of a liposomal formulation is feasible.

In summary, our data indicate that $\mathrm{LH}$ alone possessed antitumor activity against murine colon cancer in a CT26 colon cancer xenograft model. Moreover, the combination of honokiol with DDP enhanced the antitumor activity, and that the enhanced antitumor efficacy noted in vivo may partially result from the increased induction of apoptosis and the enhanced inhibition of angiogenesis in the combined treatment. The present findings may be of significance to the further exploration of the potential applications of $\mathrm{LH}$ alone or as part of the combined approach in the treatment of colon carcinoma.

\section{References}

1. Arriagada R, Bergman B, Dunant A, et al: Cisplatin-based adjuvant chemotherapy in patients with completely resected non-small-cell lung cancer. N Engl J Med 350: 351-360, 2004.

2. Olaussen KA, Dunant A, Fouret P, et al: DNA repair by ERCC1 in non-small-cell lung cancer and cisplatin-based adjuvant chemotherapy. N Engl J Med 355: 983-991, 2006.

3. Perez RP: Cellular and molecular determinants of cisplatin resistance. Eur J Cancer 34: 1535-1542, 1998 
4. Rossi A, Maione P and Gridelli C: Safety profile of platinumbased chemotherapy in the treatment of advanced non-small cell lung cancer in elderly patients. Expert Opin Drug Saf 4: 1051-1067, 2005.

5. Perrotta C, Bizzozero L, Falcone S, et al: Nitric oxide boosts chemoimmunotherapy via inhibition of acid sphingomyelinase in a mouse model of melanoma. Cancer Res 67: 7559-7564, 2007.

6. Bai X, Cerimele F, Ushio-Fukai M, et al: Honokiol, a small molecular weight natural product, inhibits angiogenesis in vitro and tumor growth in vivo. J Biol Chem 278: 35501-35507, 2003.

7. Hibasami H, Achiwa Y, Katsuzaki H, et al: Honokiol induces apoptosis in human lymphoid leukemia Molt $4 \mathrm{~B}$ cells. Int $\mathrm{J} \mathrm{Mo}$ Med 2: 671-673, 1998

8. Wang T, Chen F, Chen Z, et al: Honokiol induces apoptosis through p53-independent pathway in human colorectal cell line RKO. World J Gastroenterol 10: 2205-2208, 2004.

9. Yang SE, Hsieh MT, Tsai TH and Hsu SL: Down-modulation of Bcl-XL, release of cytochrome $\mathrm{c}$ and sequential activation of caspases during honokiol-induced apoptosis in human squamous lung cancer $\mathrm{CH} 27$ cells. Biochem Pharmacol 63: $1641-1651,2002$

10. Reimer CL, Agata N, Tammam JG, et al: Antineoplastic effects of chemotherapeutic agents are potentiated by NM-3, an inhibitor of angiogenesis. Cancer Res 62: 789-795, 2002.

11. Konoshima T, Kozuka M, Tokuda H, et al: Studies on inhibitors of skin tumor promotion, IX. Neolignans from Magnolia officinalis. J Nat Prod 54: 816-822, 1991.

12. Jin ZJ: Addition in drug combination. Acta Pharmacol Sin 1 70-76, 1980.

13. Malaguarnera L: Implications of apoptosis regulators in tumorigenesis. Cancer Metastasis Rev 23: 367-387, 2004.

14. Ferreira CG, Epping M, Kruyt FA and Giaccone G: Apoptosis: target of cancer therapy. Clin Cancer Res 8: 2024-2034, 2002.

15. Zupi G, Scarsella M, Semple SC, Mottolese M, Natali PG and Leonetti C: Antitumor efficacy of bcl-2 and c-myc antisense oligonucleotides in combination with cisplatin in human melanoma xenografts: relevance of the administration sequence. Clin Cancer Res 11: 1990-1998, 2005

16. Bourhis J, Rivera F, Mesia R, et al: Phase I/II study of cetuximab in combination with cisplatin or carboplatin and fluorouracil in patients with recurrent or metastatic squamous cell carcinoma of the head and neck. J Clin Oncol 24: 2866-2872, 2006.

17. Van Antwerp DJ, Martin SJ, Kafri T, Green DR and Verma IM: Suppression of TNF-alpha-induced apoptosis by NF-kappaB. Science 274: 787-789, 1996.

18. Hsu H, Shu HB, Pan MG and Goeddel DV: TRADD-TRAF2 and TRADD-FADD interactions define two distinct TNF receptor 1 signal transduction pathways. Cell 84: 299-308, 1996.
19. Deng Y, Ren X, Yang L, Lin Y and Wu X: A JNK-dependent pathway is required for TNFalpha-induced apoptosis. Cell 115: 61-70, 2003.

20. Weitsman GE, Ravid A, Liberman UA and Koren R: Vitamin D enhances caspase-dependent and -independent TNFalphainduced breast cancer cell death: the role of reactive oxygen species and mitochondria. Int J Cancer 106: 178-186, 2003.

21. Lüschen $S$, Scherer $G$, Ussat $S$, Ungefroren $H$ and Adam-Klages S: Inhibition of p38 mitogen-activated protein kinase reduces TNF-induced activation of NF-kappaB, elicits caspase activity, and enhances cytotoxicity. Exp Cell Res 293: 196-206, 2004

22. Nio Y, Zighelboim J, Berek J and Bonavida B: Cycloheximideinduced modulation of TNF-mediated cytotoxicity in sensitive and resistant ovarian tumor cells. Cancer Chemother Pharmacol 26: 1-8, 1990.

23. Sasaki H, Sheng Y, Kotsuji F and Tsang BK: Down-regulation of X-linked inhibitor of apoptosis protein induces apoptosis in chemoresistant human ovarian cancer cells. Cancer Res 60 5659-5666, 2000.

24. Yoshikawa A, Saura R, Matsubara $T$ and Mizuno K: A mechanism of cisplatin action: antineoplastic effect through inhibition of neovascularization. Kobe J Med Sci 43: 109-120, 1997.

25. Ahn KS, Sethi G, Shishodia S, Sung B, Arbiser JL and Aggarwal BB: Honokiol potentiates apoptosis, suppresses osteoclastogenesis, and inhibits invasion through modulation of nuclear factor-kappaB activation pathway. Mol Cancer Res 4: 621-633, 2006

26 Senior JH: Fate and behavior of liposomes in vivo: a review of controlling factors. Crit Rev Ther Drug Carrier Syst 3: 123-193, 1987.

27. Ranade VV: Drug delivery systems. 1. site-specific drug delivery using liposomes as carriers. J Clin Pharmacol 29: 685-694, 1989.

28. Rahman YE, Hanson WR, Bharucha J, Ainsworth EJ and Jaroslow BN: Mechanisms of reduction of antitumor drug toxicity of liposome encapsulation. Ann N Y Acad Sci 308: 325-341, 1978.

29. Webb MS, Harasym TO, Masin D, Bally MB and Mayer LD Sphingomyelin-cholesterol liposomes significantly enhance the pharmacokinetic and therapeutic properties of vincristine in murine and human tumour models. $\mathrm{Br} \mathrm{J}$ Cancer 72 : 896-904, 1995

30. Yuan F, Dellian M, Fukumura D, et al: Vascular permeability in a human tumor xenograft: molecular size dependence and cutoff size. Cancer Res 55: 3752-3756, 1995.

31. Gabizon AA: Pegylated liposomal doxorubicin: metamorphosis of an old drug into a new form of chemotherapy. Cancer Invest 19: 424-436, 2001. 